\title{
Gene disruption of ribosomal protein L5 (RPL5) decreased the sensitivity of CHO-K1 cells to uncoupler carbonylcyanide-3- chlorophenylhydrazone
}

\author{
Makoto Araki ${ }^{1}$, Takuya Ishibashi ${ }^{2}$, Masahiro Munesue ${ }^{3, \S}$, Kazuaki Ohashi ${ }^{4}$, \\ Yoshitaka Nobukuni ${ }^{5}$, Masatomo Maeda ${ }^{6, *}$ \\ ${ }^{1}$ Meiji Pharmaceutical University, Tokyo, Japan; \\ ${ }^{2}$ Ritsumeikan-Global Innovation Research Organization, Ritsumeikan University, Kyoto, Japan; \\ ${ }^{3}$ Graduate School of Pharmaceutical Sciences, Osaka University, Suita, Osaka, Japan; \\ ${ }^{4}$ School of Pharmacy, Iwate Medical University, Iwate, Japan; \\ ${ }^{5}$ Research Institute for Radiation Biology and Medicine, Hiroshima University, Hiroshima, Japan; \\ ${ }^{6}$ Faculty of Pharmaceutical Sciences, Niigata University of Pharmacy and Applied Life Sciences, Niigata, Japan.
}

\begin{abstract}
Summary Protonophoric uncoupler carbonylcyanide-3-chlorophenylhydrazone (CCCP) decreases the proton motive force $(\triangle \mathrm{P})$ of the mitochondrial inner membrane and results in inhibition of oxidative phosphorylation. In this study, a CCCP-resistant clone was isolated from a random gene trap insertional mutant library of Chinese hamster ovary (CHO)-K1 cells which was constructed by infecting a retrovirus vector, ROSABgeo. Although we expected the isolation of the mutants defective in nuclear genes responsible for mitochondrial functions, the disrupted gene of the isolated mutant that we named $R 1$ cells was identified as one of the alleles for ribosomal protein 5 of large subunit (RPL5). The R1 cells express as much as $80 \%$ RPL5 protein compared with the parental CHO-K1 cells, possibly due to enhanced transcription from a remaining wild-type $R P L 5$ allele in $R 1$ cells. Furthermore, the protein amount is not decreased by CCCP in R1 cells, in contrast to its clear reduction by CCCP in parental cells. Since mutations of RPL5 and other ribosomal proteins are responsible for the ribosomopathies and cancer, the present mutant may be a useful cellular model of such human diseases from a viewpoint of energy metabolism as well as a tool for the study of ribosome biogenesis and extra-ribosomal function of the RPL5 protein.
\end{abstract}

Keywords: Ribosomopathies, Diamond-Blackfan anemia, random gene trap mutagenesis, uncoupler, mitochondria

\section{Introduction}

Mitochondria play vital roles in aerobic energy production and homeostasis in living cells (1). The respiratory chain, through which electrons are transferred, produces an electrochemical gradient of $\mathrm{H}^{+}$ across the mitochondrial inner membrane. This proton motive force $(\Delta \mathrm{P})$ drives the synthesis of cellular energy currency, ATP, by ATP synthase (2). Furthermore, more

\footnotetext{
${ }^{\S}$ Present Address: Santen Pharmaceutical Co., Ltd., Japan. *Address correspondence to:

Dr. Masatomo Maeda. Laboratory of Gene Therapy, Faculty of Pharmaceutical Sciences, Niigata University of Pharmacy and Applied Life Sciences, Niigata 956-8603, Japan.

E-mail:mmaeda@nupals.ac.jp
}

than 1,200 nuclear genes are believed to maintain and regulate the mitochondrial structure and function (3), although mammalian mitochondrial DNA retains 37 genes (1). Thus, it is not surprising that mitochondrial dysfunction would be induced by various mutations in both the nuclear and mitochondrial genomes (4), which results in health problems $(1,3)$.

Uncouplers are compounds that translocate $\mathrm{H}^{+}$ across a membrane passively and dissipate $\Delta \mathrm{P}(5)$. Carbonylcyanide-3-chlorophenylhydrazone (CCCP) is an example of such protonophoric uncouplers (6), and is known to be an apoptotic inducer (7). Inhibition of oxidative phosphorylation due to a decrease in $\Delta \mathrm{P}$ would induce reactive oxygen species, and finally lead to apoptosis of the cells $(8)$. In this study, we attempted 
to isolate CCCP-resistant clones of mammalian cultured cells since mutations of nuclear genes would reduce the toxic effect of CCCP. Phenotypic studies of the mutants will not only clarify gene functions but also give an idea of treatments for mitochondrial or mitochondriallike disorders (3).

In this study, we adopted gene trap mutagenesis, a technique generating loss-of-function mutations randomly (9). We screened a random gene trap insertional mutant library of Chinese hamster ovary (CHO)-K1 cells (10) constructed by infection with the retrovirus gene trap vector ROSA $\beta$ geo (11). The isolated CCCP-resistant clone exhibited haploinsufficiency of the gene for ribosomal protein 5 of large subunit (RPL5). We characterized this clone, and discussed its properties from the viewpoints of energy metabolism and roles of RPL5.

\section{Materials and Methods}

\subsection{Cell culture}

CHO-K1 cells (12) and the random gene trap insertional mutant library (10) were cultured in Ham's F-12 medium (Sigma, MO, USA) supplemented with $10 \%$ $(\mathrm{v} / \mathrm{v})$ fetal bovine serum (Biowest, Nuaille France) in a $\mathrm{CO}_{2}$ incubator at $37^{\circ} \mathrm{C}$. CCCP (Nacalai, Kyoto, Japan) dissolved in dimethyl sulfoxide (DMSO) $(10 \mathrm{mM})$ was added at the indicated concentration in the medium. Control culture medium without CCCP contained a similar amount of DMSO. The cell cycle was analyzed with a Muse Cell Analyzer (Merck Millipore, MA, USA) according to the manufacturer's protocol using a Muse Cell Cycle Kit (13). The sensitivity to CCCP and colchicine (Calbiochem, CA, USA) was examined by colony formation at $37^{\circ} \mathrm{C}(14)$.

\subsection{Identification of the disrupted gene}

Total RNA of R1 cells was prepared with an RNeasy Mini Kit (Qiagen, Hilden, Germany) according to the manufacturer's protocol, and reverse transcribed with phosphorylated primer LacZr 005 and single-stranded cDNA was circulalized. The product was subjected to polymerase chain reaction (PCR) with primer pairs LacZf 003/LacZr 002 and LacZf 004/LacZr 001 (Table 1) and GoTaq ${ }^{\circledR}$ (Promega, WI, USA) successively; preheating $\left(96^{\circ} \mathrm{C}, 5 \mathrm{~min}\right)$, followed by 30 cycles of denaturation $\left(96^{\circ} \mathrm{C}, 0.5 \mathrm{~min}\right)$, annealing $\left(55^{\circ} \mathrm{C}, 0.5\right.$ min), and extension $\left(72^{\circ} \mathrm{C}, 1 \mathrm{~min}\right)$, and then postincubation $\left(72^{\circ} \mathrm{C}, 7 \mathrm{~min}\right)$ in a GeneAmp PCR System 2400 (PerkinElmer, MA, USA). The DNA fragments separated by $2 \%$ agarose gel-electrophoresis were ligated into the pGEM-T easy vector (Promega) and then introduced into Escherichia coli TOP-10F'. The DNA sequence was determined by the dideoxy chaintermination method with Ampli Taq (Roche, Basel,
Table 1. List of PCR primers used in this study

\begin{tabular}{ll}
\hline LacZr 001: & 5'-TTCCCAGTCACGACGTTGTA-3' \\
LacZr 002: & 5'-GTGCTGCAAGGCGATTAAGT-3' \\
LacZf 003: & 5'-GTTGATGAAAGCTGGCTACA-3' \\
LacZf 004: & 5'-TGATGGCGTTAACTCGGCGT-3' \\
LacZr 005: & 5'-ATGCGCTCAGGTCAAATTCA-3' \\
& \\
P1: & 5'-GACGGTGTCTGTTCCGCAGGATG-3' \\
P2: & 5'-GACCTTTAGCTCTCAGCAGCCCG-3' \\
P3: & 5'-AGGAAGCGGTCAGCCCATTCG-3' \\
RPL5f: & 5'-TGTCTGTTCCGCAGGATGGG-3' \\
RPL5r: & 5'-CAGTTTTACCCTCTCGCCGCC-3' \\
actin-f: & 5'-AGAGCTATGAGCTGCCTGATG-3' \\
actin-r: & 5'-CGTGGATGCCACAGGATTC-3' \\
T7 primer: & 5'-TAATACGACTCACTATA-3' \\
M13R primer: & 5'-GGAAACAGCTATGACCATG-3' \\
\hline
\end{tabular}

Switzerland) and a sequence primer (T7 or M13R) (15). The molecular biological techniques were performed by published methods (16).

\subsection{SDS-polyacrylamide gel-electrophoresis and Western blotting}

Cells treated with or without $5 \mu \mathrm{M}$ CCCP for $24 \mathrm{~h}$ were collected in $1 \mathrm{~mL}\left(3 \times 10^{5}\right.$ cells $)$ of ice-cold 20 $\mathrm{mM}$ tris(hydroxymethyl)aminomethane (Tris)- $\mathrm{HCl}$ ( $\mathrm{pH}$ 7.5), $150 \mathrm{mM} \mathrm{NaCl}, 2 \mathrm{mM}$ ethylendiaminetetraacetic acid, $10 \mu \mathrm{g} / \mu \mathrm{L}$ leupeptin, $10 \mu \mathrm{g} / \mu \mathrm{L}$ pepstatin (TNE) containing $1 \%(\mathrm{w} / \mathrm{v}) \mathrm{NP}-40$ and then kept on ice for 30 min. After sheering of the suspension 10 times through a $25 \mathrm{G}$ needle, a supernatant $(12,000 \times \mathrm{g}, 30 \mathrm{~min})$ was obtained. The resulting cell extract was subjected to sodium dodecyl sulfate (SDS)-polyacrylamide gelelectrophoresis (17), and then electro-blotted onto a Hybond-P PVDF membrane (GE Healthcare, IL, USA) (18).

The membrane was blocked overnight at $4{ }^{\circ} \mathrm{C}$ with $10 \mathrm{mM}$ sodium phosphate buffer ( $\mathrm{pH} 7.2), 137 \mathrm{mM}$ $\mathrm{NaCl}, 3 \mathrm{mM} \mathrm{KCl}$ (PBS) containing $0.1 \%$ (v/v) Tween-20 and 3\% (w/v) skim milk (Wako, Osaka, Japan). Rabbit and mouse polyclonal antibodies recognizing human RPL5 (Abcam, Cambridge, UK) and glyceraldehyde3-phosphate dehydrogenase (GAPDH) (Wako), respectively, were reacted for $1 \mathrm{~h}$ as the first antibodies [ $\times 5000$ dilution in $25 \mathrm{mM}$ Tris- $\mathrm{HCl}(\mathrm{pH} 7.6), 150 \mathrm{mM}$ $\mathrm{NaCl}$ containing $0.1 \%(\mathrm{w} / \mathrm{w})$ Tween-20 (TBS-T)]. The membrane was washed in TBS-T for 5 min (3 times). Horseradish peroxidase-linked goat anti-rabbit and antimouse immunoglobulins ( $\times 5000$ diluted) (Jackson, PA, USA) were used as the second antibodies with ECL Prime Western Blotting Detection Reagent (GE Healthcare). The RPL5 and GAPDH were visualized with a LAS-3000 (Fuji Film, Tokyo, Japan). To detect GAPDH the membrane was re-probed after analysis of RPL5 (19). 


\subsection{Quantitative analysis of RPL5 $m R N A$}

Total RNA of R1 and CHO-K1 cells was reverse transcribed with a PrimeScript RT reagent kit (TaKaRa, Shiga, Japan) with a random 6 mer primer. Real time PCR was performed on an Eco Real Time PCR System (Illumina, CA, USA) and SYBR Premix Ex Taq II (TaKaRa) with primer pair RPL5f/RPL5r or $\beta$ Actin-f/ $\beta$ Actin-r. The data were analyzed by the $\Delta \Delta \mathrm{Ct}$ method.

\subsection{Staining of mitochondria}

Cells cultured for $24 \mathrm{~h}$ on poly-L-lysine-coated chamber slides (Iwaki, Shizuoka, Japan) were further treated with $5 \mu \mathrm{M}$ CCCP or DMSO for $8 \mathrm{~h}$. The treated cells were incubated for $15 \mathrm{~min}$ in medium containing 100 nM MitoTracker Red CMX Ros (Molecular Probes, OR, USA) and then washed with fresh medium. The cells were fixed with $4 \%$ formaldehyde. The fluorescence of fixed cells was detected under an Olympus confocal microscopy system FV1000 (IX81), the excitation and detection conditions were adjusted to the channel for AlexaFlour 568 (Invitrogen, CA, USA).

\subsection{Chemicals}

Restriction enzymes were purchased from NEB (MA, USA) and Toyobo (Osaka, Japan). RNaseH, T4 DNA ligase (Ligation Kit Ver.2.1), and a 5'-Full RACE Core Set were obtained from TaKaRa. Oligonucleotides were supplied by Invitrogen. A GENECLEAN III Kit was obtained from BIO101 (CA, USA). All other chemicals used were of the highest grade commercially available.

\section{Results}

\subsection{Isolation of a CCCP-resistant clone}

Since more than $3 \mu \mathrm{M}$ CCCP inhibited the colony formation of parent $\mathrm{CHO}-\mathrm{K} 1$ cells, we selected the CCCP-resistant cells in the presence of $5 \mu \mathrm{M}$ CCCP. Three colonies were formed from a culture of $3.4 \times 10^{7}$ cells after 14 days. However, only one clone showed partial resistance to CCCP and other two clones did not grow in the presence of CCCP after colony isolation. We named this clone R1, and further characterized its properties and identified a disrupted gene.

$\mathrm{R} 1$ cells formed colonies in the presence of $3 \mu \mathrm{M}$ CCCP. However, the resistance phenotype was not significant, colony formation decreasing in parallel with the wild type and not being detected under the isolation conditions $(5 \mu \mathrm{M})$ (Figure 1A). Interestingly, R1 cells that had been treated with $5 \mu \mathrm{M}$ CCCP in Figure 1A formed a significant number of colonies (as much as 700 colonies) in the fresh medium without CCCP while none grew on the plate of wild type. Thus, the R1 cells were still resistant and alive in the presence of $5 \mu \mathrm{M}$
CCCP without growth although wild type CHO-K1 cells were completely killed under the same conditions.

Furthermore, as evident from Figure 1B, the R1 clone was not resistant to colchicine, suggesting that the resistance of R1 cells to CCCP could not be ascribed to the multidrug resistance phenotype (20).

\subsection{Identification of a disrupted gene}

We first cloned the exon moiety of the disrupted gene preceding the inserted $\beta g e o$. The 5'-rapid amplification of cDNA ends technique amplified a 290 bp DNA fragment which had an upstream additional sequence, as indicated by the capital letters in the "Trapped sequence" in Figure 2A. This sequence corresponds to a component of a large 60S ribosomal subunit, RPL5, essentially identical to those of Chinese hamster, human and rodents. All the published sequence data except pROSA $\beta$ geo (Figure 2A) were obtained from National Center for Biotechnology Information (https://www. ncbi.nlm.nih.gov, accessed September 30, 2016).
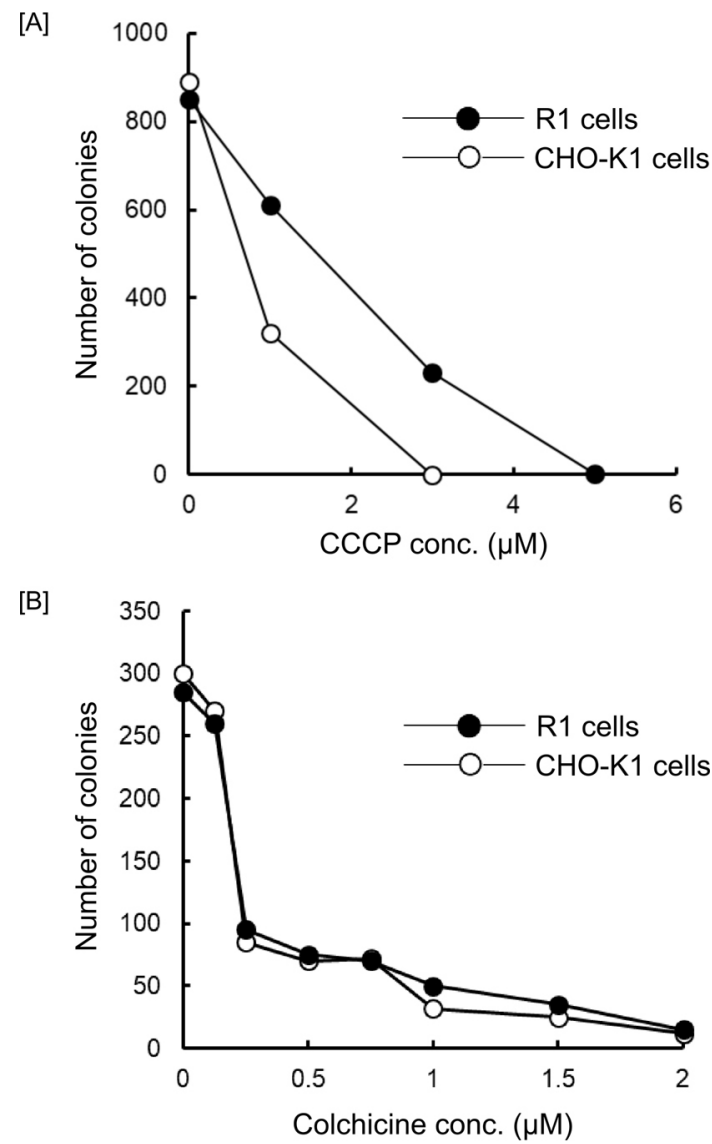

Figure 1. Colony formation of the CCCP-resistant clone in the presence of CCCP and colchicine. [A] Cells $\left(10^{4}\right.$ cells $)$ were seeded in $6 \mathrm{~cm}$ diameter dishes. CCCP was added on the following day. Then the cells further cultured for 9 days were stained and colonies were counted. Each point represents the mean \pm standard deviation of duplicate sample dishes. [B] Cells $(2,000$ cells $)$ were seeded in each well of a 24 wellplate. Various concentrations of colchicine were added at $5 \mathrm{~h}$ after inoculation. On the 6th day, the cells were stained and colonies were counted. 
To demonstrate that the gene disruption event had truly occurred in the RPL5 gene, we constructed a PCR primer pair, $\mathrm{P} 1 / \mathrm{P} 2$, to amplify the mRNA between exon 1 and exon 8 transcribed from a normal allele of RPL5. A primer pair, P1/P3, was used to amplify the fused mRNA from the disrupted allele (Figure $2 \mathrm{~B}$ ). As shown in Figure $2 \mathrm{C}$, only primer pair $\mathrm{P} 1 / \mathrm{P} 2$ gave the amplified band from CHO-K1 cells. However, both the $\mathrm{P} 1 / \mathrm{P} 2$ and $\mathrm{P} 1 / \mathrm{P} 3$ primer pairs gave products from R1 cells corresponding to the estimated sizes (919 bp and $3910 \mathrm{bp}$, respectively). It must be noted that the $\mathrm{CHO}$ genome project suggests the presence of a single-copy of RPL5 gene (Gene ID 100770250) in CHO-K1 cell (21). These results suggest that one of the two RPL5 genes could be disrupted in R1 cells.

Since exon 1 of the RPL5 gene in human and rodent ends at the initiation codon, it is suggested that the retrovirus vector was inserted into intron 1 of one of the RPL5 genes of R1 cells (Figure 2B and Figures 3A and $3 \mathrm{~B})$. Furthermore, it was clear that the splicing donor site "gt" was located next to the initiation codon of the RPL5 gene in R1 cells, and that the splicing acceptor

$[\mathrm{A}]$

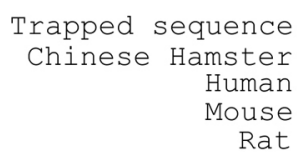
Trapped sequence Chinese Hamster Human Mouse
Rat

Trapped sequence Chinese Hamster Human Mouse Rat

Trapped sequence

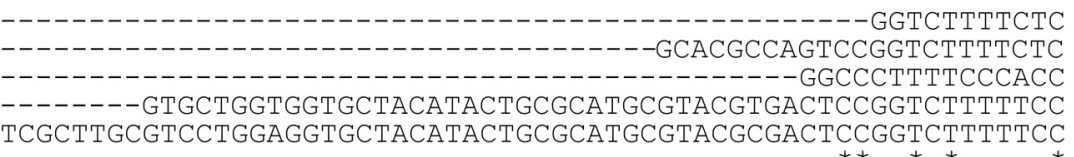

CCCT-AGCGCCGCCGGGCCCGCAGGTCTGTGTGGAGCCGCGGTTGCGGGTGTCTGTTCCG CCCT-AGCGCCGCCGGGCCCGCAGGTCTGTGTGGAGCCGCGGTTGCGGGTGTCTGTTCCG CCCT-AGCGCCGCTGGGCCTGCAGGTCTCTGTCGAGCAGCGGACGCCGGTCTCTGTTCCG CCCTTAGCGCGGCCGACTCTGCAGGTCTGCGTGGAGCCGCGGTTGCGGGTCTCTATTCCG CCCT-AGCGCGGCCGACTCTGCAGGTCTGCGTGGAGCCGCAGTTGCGGGTCTCTGTTCCG

CAGGATGctcgcggttgaggacaaactcttcgcggtctttccagtggggatcgacggtat CAGGATG

CAGGATG

CAGGATG

CAGGATG

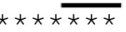

cgataagcttgatgatctgtgacatggcggatcccgtcgttttacaacgtcg
[B]

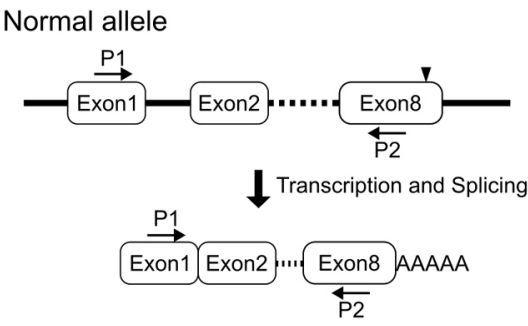

Disrupted allele

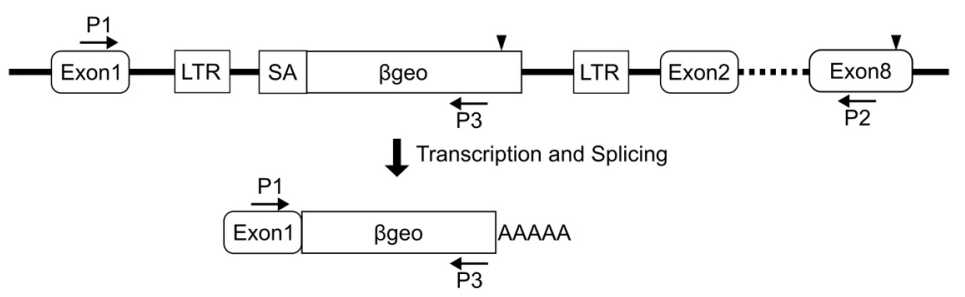

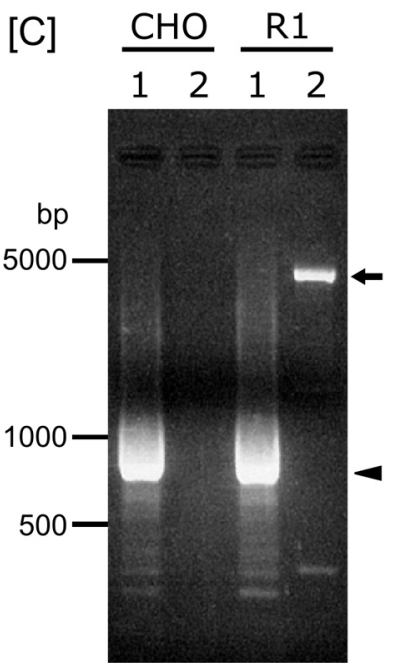

Figure 2. Identification of the disrupted gene. [A] The trapped sequence (capital letters) was aligned with the corresponding part of RPL5 cDNA of Chinese hamster, human, mouse and rat (XM_007642956, NM_000969, NM_016980 and NM_031099, respectively). The initiation codon is underlined. Lowercase letters and asterisks indicated the sequence derived from the pROSARgeo vector [SA-betageo (Addgene plasmid \#21709); http://n2t/addgene:21709 (accessed July 15, 2016)] and the conserved nucleotide residues, respectively. [B] Gene trap event on the RPL5 gene in R1 cells is schematically represented. The cDNA sequence (XM 007642956) clearly indicated that the exons of Chinese hamster might be separated by 7 introns similar to those of human and mouse (NG_011779 and NC_000071, respectively). The gene trap vector was suggested to be inserted into intron 1 (see legend to Figure $3 \mathrm{~B}$ ). Thus, the primer pair P1/P2 amplified the cDNA derived from a normal transcript while the primer pair P1/P3 gave a product from a fusion transcript of exon 1 with $\beta$ geo. Only P1/P2, i.e. not P1/P3, amplified the cDNA from CHO-K1 cells. Closed triangle $(\boldsymbol{\nabla})$ indicates the polyA signal. [C] Total cellular RNA was reverse-transcribed using random oligonucleotide primers. The cDNA derived from a transcript of the RPL5 gene was amplified by means of PCR with primer pair P1/P2 (Sample 1) or P1/P3 (Sample 2). The annealing sites of primers and primer sequences are shown in [B] and Table 1, respectively. The PCR products were analyzed by $1 \%$ agarose gel electrophoresis (16). The arrow and arrowhead indicate those derived from the fusion gene and the intact one, respectively. 
site "ag" in the vector sequence was used (Figure 3B), consisting with the finding that a retrovirus vector tends to insert into the 5 ' portion of the gene $(9)$.

\subsection{Properties of R1 cells}

We next determined the expression levels of RPL5 protein and mRNA under the steady state culture conditions by Western blotting analysis and quantitative PCR, respectively. In the absence of CCCP, the amount of RPL5 protein in R1 cells was about $80 \%$ of that in the parental CHO-K1 cells in spite of that the normal RPL 5 gene in the former cells comprised one half of the latter (Figure 4A, lanes 1 and 3). Such observation could be partly explained by the increased transcription from the remaining wild-type allele (22). Actually, the mRNA

$[\mathrm{A}]$

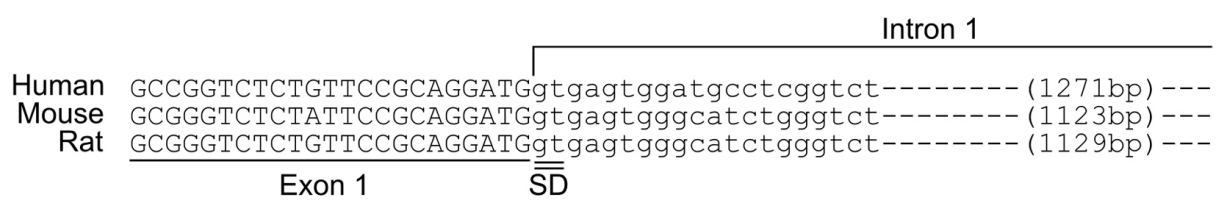
Exon $1 \quad$ SD

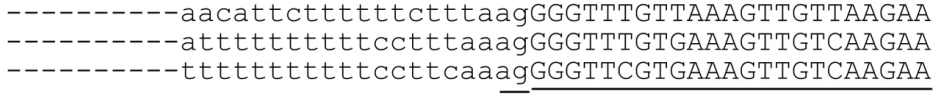

$\overline{\overline{S A}} \quad$ Exon 2

[B]

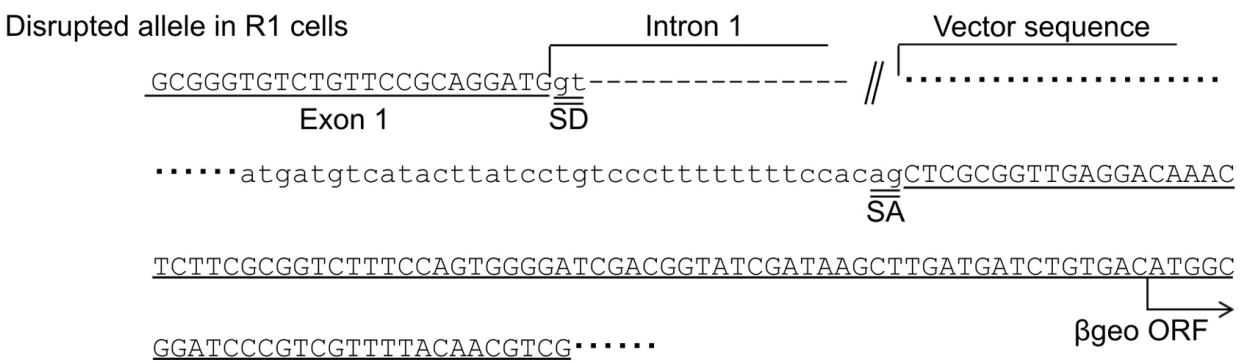

Figure 3. Comparison of the genomic sequences and splicing sites of RPL5 of various species. [A] The nucleotide sequences of downstream of exon 1, upstream of exon 2 and both ends of intron 1 for the human (NG_011779) and rodents (NC_000071 for mouse and NC 005113 for rat) RPL5 genes were aligned. The nucleotide residues of introns are indicated by lowercase letters. Splicing donor site "gt" and acceptor site "ag" (32) are double-underlined, and denoted by SD and SA, respectively. [B] The gene disruption event that occurred in the R1 cell is explained: the retrovirus vector was inserted into intron 1 of the RPL5 gene. The splicing donor site (SD) of intron 1 of the RPL5 gene and the splicing acceptor site (SA) of the $\beta g e o$ gene in the retrovirus vector are indicated by double underlines. Canonical splicing (32) occurred during transcription of the fusion gene, and both underlined sequences in capital letters were connected, as is evident from the trapped sequence shown in Figure $2 \mathrm{~A}$.
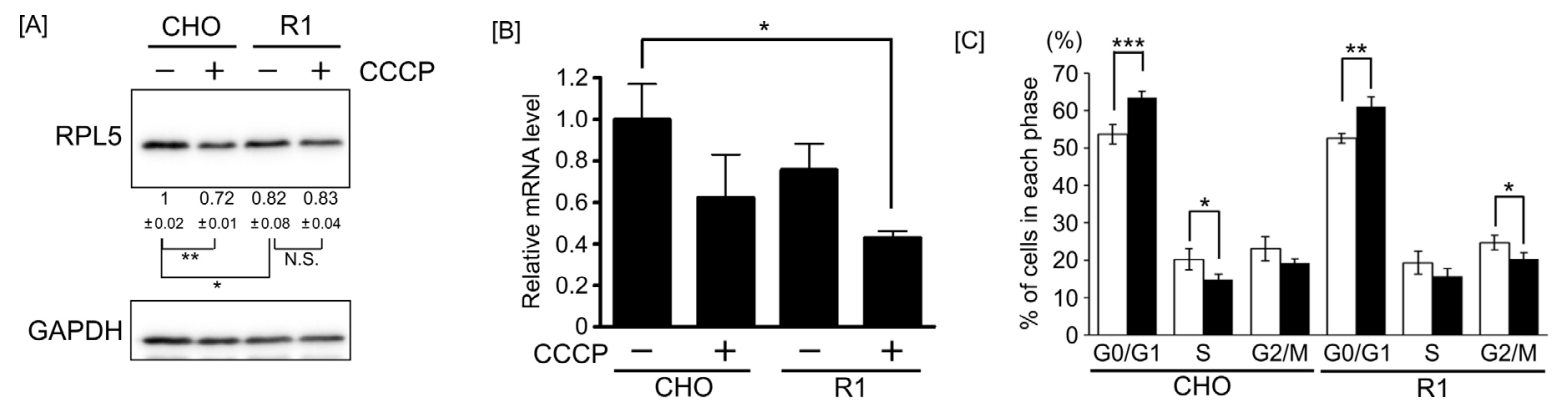

Figure 4. Expression levels of protein and mRNA for RPL5, and cell cycle analyses. [A] Cell lysates (15 $\mu \mathrm{g})$ were separated by SDS-polyacrylamide gel-electrophoresis. RPL5 and GAPDH were detected by means of Western blotting with anti-RPL5 (upper) and anti-GAPDH (lower) antibodies, respectively. The numerical values at the bottom of blot are the relative amounts of RPL5 protein normalized as to those of housekeeping GAPDH protein. The standard deviations of band intensity $(n=3)$ were determined with ImageJ software [http://imagej.nih.gov/ij/ (accessed August 20, 2019)]. The data were analyzed by means of Tukey-Kramer test. ${ }^{*} p$-values $<0.05$ and ${ }^{* *} p<0.01$ were considered statistically significant. N.S.; Not Significant. This experiment was repeated twice and a similar tendency was observed. [B] Total cellular RNA was prepared, and the mRNA expression level of RPL5 was determined by real-time PCR. The expression level was normalized as to that of $\beta$-actin mRNA. The results of triplicate samples are presented as mean values \pm standard deviation $(n=3)$. Tukey-Kramer test was performed as in [A]. * ( $p$-values $<0.05)$. [C] The R1 and CHO-K1 cells that had been cultured with (closed bar) or without $5 \mu \mathrm{M} \mathrm{CCCP} \mathrm{(open}$ bar) for $24 \mathrm{~h}$ were analyzed. The percentage of each phase in the culture was shown. Each bar represents the mean \pm standard deviation of three independent experiments. The data were analyzed by means of 2 -tailed unpaired Student's $t$-test. * $p$-values $<0.05$, $* * p<0.01$ and $* * * p<0.001$ were considered statistically significant. 


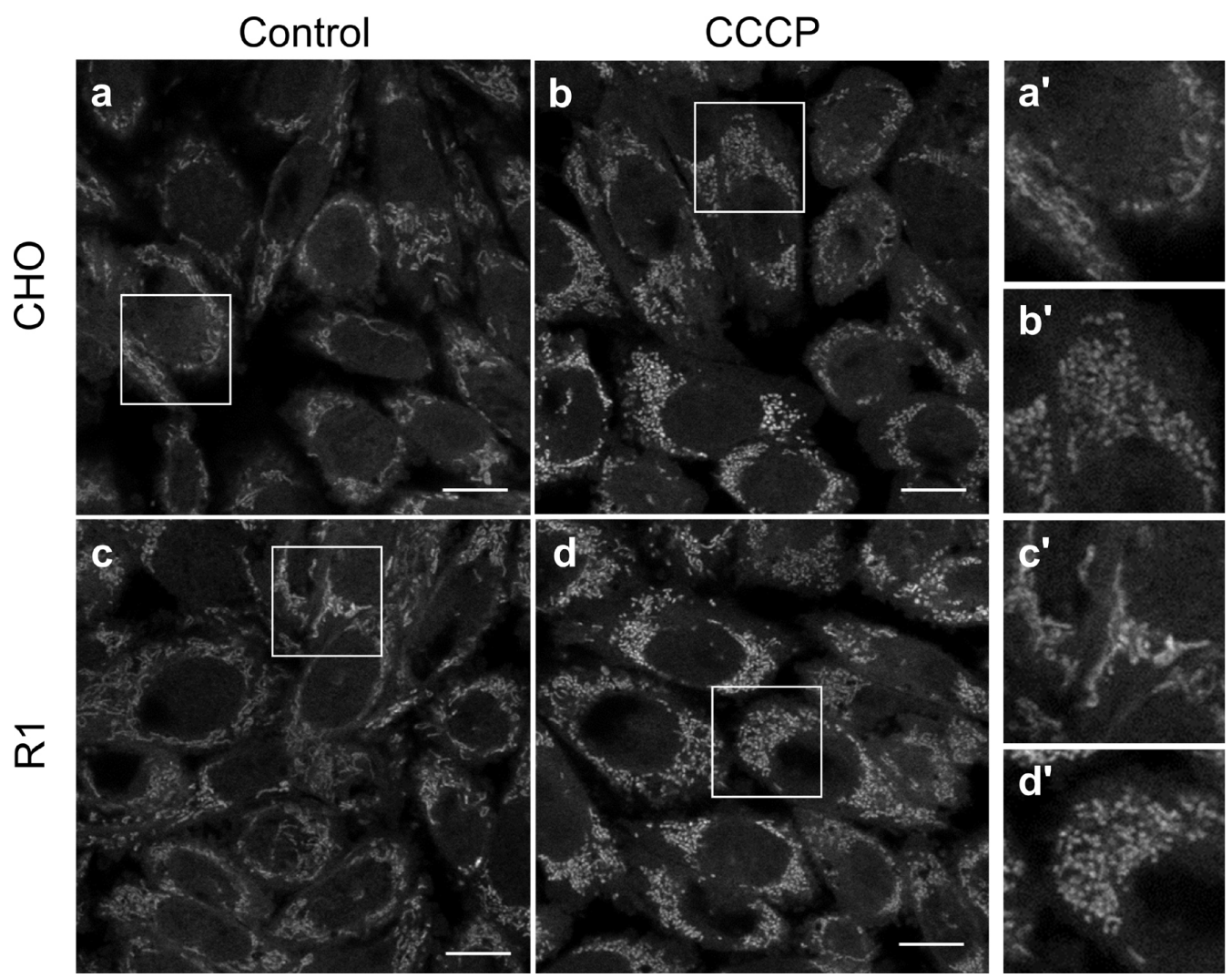

Figure 5. Morphology of mitochondria in R1 and CHO-K1 cells treated with or without CCCP. Cells cultured on polyL-lysine-coated chamber slides for $24 \mathrm{~h}$ were treated with or without $5 \mu \mathrm{M} \mathrm{CCCP}$ for $8 \mathrm{~h}$, and then further incubated in fresh medium containing $100 \mathrm{nM}$ MitoTracker Red CMX Ros without CCCP. The fluorescence of the fixed cells was observed under a confocal microscope. Magnification bars, $10 \mu \mathrm{m}$. Three independent experiments gave essentially the same results. The boxed areas in a - d were enlarged ( $\mathrm{a}^{\prime}-\mathrm{d}^{\prime}$, respectively). We further examined mitochondrial images in the presence of CCCP for $24 \mathrm{~h}$. However, they were indistinguishable from those with $8 \mathrm{~h}$ (not shown). The mitochondrial numbers in the presence of CCCP were calculated for total 135 cells in three different images from CHO-K1 and R1 cells, respectively. Although relative amounts of R1 cells were about 1.52 -fold of those of CHO-K1 cells, the difference between CHO-K1 and R1 cells was not significant $(p$-value $=0.07)$.

level of R1 cells was more than half of CHO-K1 cells (Figure 4B, without CCCP), as was suggested by protein levels at steady state being primarily determined by mRNA levels (23).

The mRNA levels of both R1 and CHO-K1 cells further decreased in the presence of CCCP (Figure 4B). Concomitantly, the amount of RPL5 protein decreased in CHO-K1 cells in the presence of CCCP (Figure 4A, lanes 1 and 2). However, it remained constant in R1 cells (Figure 4A, lanes 3 and 4). It seems likely that the stability of RPL5 protein may increase in R1 cells upon adding CCCP to save energy required for transcriptional and translational processes.

Cell cycle analyses demonstrated that the distribution of cells in the G0/G1, S and G2/M phases was essentially the same between R1 and CHO-K1 cells in the presence and absence of CCCP although the presence of CCCP for $24 \mathrm{~h}$ slightly increased the population in $\mathrm{G} 0 / \mathrm{G} 1$ for both cell types. Thus, the effect of CCCP on cell cycle progression was essentially the same in resistant and sensitive cells (Figure 4C).

We further observed mitochondrial images of both $\mathrm{R} 1$ and CHO-K1 cells in the presence of CCCP for $8 \mathrm{~h}$ on staining with a fluorescent organelle marker,
MitoTracker Red CMX Ros (Figure 5). Both cells without CCCP showed essentially the same filamentous mitochondrial structure (Figures $5 \mathrm{a}$ and $5 \mathrm{c}$ ). In the presence of CCCP, mitochondria became shortened and vesicular (Figures $5 \mathrm{~b}$ and $5 \mathrm{~d}$ ) as indicated by the enlarged figures (Figures 5a'-5d'). However, we could not detect significant difference between mitochondrial morphology and numbers for R1 and CHO-K1 cells in the presence of CCCP (Figure 5).

\section{Discussion}

The isolated R1 cells showed partial resistance to CCCP, which could be explained by that $\mathrm{R} 1$ cells are more resistant to a low-energy supply and survive under the energetic stress conditions in the presence of CCCP, although they would be difficult to grow under a higher concentration of CCCP due to an extremely low energy level or a toxic effect of CCCP other than uncoupling (O).

Haploinsufficiency of ribosomal proteins essential for cell growth may impair functional ribosomal biogenesis in general (24). Ribosomopathies are human disorders associated with defects of ribosomal subunit genes and ribosome biogenesis (25). A typical example 
is Diamond-Blackfan anemia: about $60-70 \%$ of the patients are heterozygotes for mutations and deletions of various ribosomal subunit genes (26). Furthermore, the genes for ribosomal protein 19 of small subunit and RPL5 are most often altered (27). If we consider that RPL5 stabilizes p53 (28) and cooperatively inactiveates c-Myc (29), such tumor suppressor functions of RPL5 are consistent with the finding that heterozygous inactivation of RPL5 associates with the most common somatic ribosomal defect in human cancer (30).

Cancer cells are known to depend on the aerobic glycolysis for continued growth and survival (31). Since our culture condition is aerobic, it seems likely that such survival of R1 cells must be supported by aerobic metabolism even without mitochondrial energetic functions under oxidative stress condition. Thus, the CCCP-resistant R1 cells with a heterozygous mutation of RPL5 will become a tool for the study of not only cellular regulatory mechanisms concerning ribosomal biogenesis and extra-ribosomal functions of the RPL5 protein but also ribosomopathies, cancer etiology and therapy.

\section{Acknowledgements}

We thank Dr. Philippe Soriano (Department of Developmental and Regenerative Biology, Icahn School of Medicine at Mt. Sinai) for kindly providing the plasmid construct, pROSA $\beta$ geo.

\section{References}

1. Kagawa Y, Cha SH, Hasegawa K, Hamamoto T, Endo H. Regulation of energy metabolism in human cells in aging and diabetes: FoF1, mtDNA, UCP, and ROS. Biochem Biophys Res Commun. 1999; 266:662-676.

2. Mitchell P. Keilin's respiratory chain concept and its chemiosmotic consequences. Science. 1979; 206:11481159.

3. Suomalainen A. Mitochondrial roles in disease: a box full of surprises. EMBO Mol Med. 2015; 7:1245-1247.

4. Angelini C, Bello L, Spinazzi M, Ferrati C. Mitochondrial disorders of the nuclear genome. Acta Myol. 2009; 28:1623.

5. Terada H. Uncouplers of oxidative phosphorylation. Environ Health Perspect. 1990; 87:213-218.

6. Newell KJ, Tannock IF. Reduction of intracellular $\mathrm{pH}$ as a possible mechanism for killing cells in acidic regions of solid tumors: effects of carbonylcyanide-3chlorophenylhydrazone. Cancer Res. 1989; 49:4477-4482.

7. Arena G, Gelmetti V, Torosantucci L, Vignone D, Lamorte G, De Rosa P, Cilia E, Jonas EA, Valente EM. PINK1 protects against cell death induced by mitochondrial depolarization, by phosphorylating Bcl$\mathrm{xL}$ and impairing its pro-apoptotic cleavage. Cell Death Differ. 2013; 20:920-930.

8. Wallace DC. Mitochondrial diseases in man and mouse. Science. 1999; 283:1482-1488.

9. Stanford WL, Cohn JB, Cordes SP. Gene-trap mutagenesis: past, present and beyond. Nature Rev
Genetics. 2001; 2:756-768.

10. Nobukuni Y, Kohno K, Miyagawa K. Gene Trap mutagenesis-based forward genetic approach reveals that the tumor suppressor OVCA1 is a component of the biosynthetic pathway of diphthamide on elongation factor 2. J Biol Chem. 2005; 280:10572-10577.

11. Friedrich G, Soriano P. Promoter traps in embryonic stem cells: a genetic screen to identify and mutate developmental genes in mice. Genes Dev. 1991; 5:15131523.

12. Maeda M, Nishijima M, Akamatsu Y, Sakakibara Y. Alteration in the characters of CDP-choline synthetase and phospholipid-choline exchange enzyme upon choline starvation in Chinese hamster ovary cells. J Biol Chem. $1985 ; 260: 5925-5930$.

13. Ushijima H, Horyozaki A, Maeda M. Anisomycininduced GATA- 6 degradation accompanying a decrease of proliferation of colorectal cancer cells. Biochem Biophys Res Commun. 2016; 478:481-485.

14. Maeda M, Ishida A, Ni L, Kobayashi A. Isolation of CHO-K 1 clones defective in cAMP-dependent proteolysis, as determined by the stability of exogenously expressed GATA-6. Biochem Biophys Res Commun. 2005; 329:140-146.

15. Sanger F, Coulson AR, Barrell BG, Smith AJH, Roe BA. Cloning in single-stranded bacteriophage as an aid to rapid DNA sequencing. J Mol Biol. 1980; 143:161-178.

16. Sambrook J, Fritsch EF, Maniatis T. Molecular Cloning: A Laboratory Manual, 2nd ed. Cold Spring Harbor Laboratory, Cold Spring Harbor. 1989.

17. Laemmli UK. Cleavage of structural proteins during the assembly of the bacteriophage T4. Nature. 1970; 227:680685.

18. Towbin H, Staehelin T, Gordon J. Electrophoretic transfer of proteins from polyacrylamide gels to nitrocellulose sheets: procedure and some applications. Proc Natl Acad Sci U S A. 1979; 76:4350-4354.

19. Mehra-Chaudhary R, Matsui H, Raghow R. Msx3 protein recruits deacetylase to down-regulate the Msx 1 promoter. Biochem J. 2001; 353:13-22.

20. Sharma RC, Inoue S, Roitelman J, Schimke RT, Simoni RD. Peptide transport by the multidrug resistance pump. J Biol Chem. 1992; 267:5731-5734.

21. Kremkow BG, Baik JY, MacDonald ML, Lee KH. CHOgenome.org 2.0: Genome resources and website updates. Biotechnol J. 2015; 10:931-938.

22. Mills EW, Green R. Ribosomopathies: There's strength in numbers. Science. 2017; 358:eaan2755.

23. Liu Y, Beyer A, Aebersold R. On the dependency of cellular protein levels on mRNA abundance. Cell. 2016; 165:535-550.

24. Teng T, Mercer CA, Thomas G, Fumagalli S. Loss of tumor suppressor RPL5/RPL11 does not induce cell cycle arrest but impedes proliferation due to reduced ribosome content and translation capacity. Mol Cell Biol. 2013; 33:4660-4671.

25. Narla A, Ebert BL. Ribosomopathies: human disorders of ribosome dysfunction. Blood. 2010; 115:3196-3205.

26. Farrar JE, Vlachos A, Atsidaftos E, Carlson-Donohoe H, Markello TC, Arceci RJ, Ellis SR, Lipton JM, Bodine DM. Ribosomal protein gene deletions in DiamondBlackfan anemia. Blood. 2011; 118:6943-6951.

27. Kuramitsu M, Sato-Otsubo A, Morio T, et al. Extensive gene deletions in Japanese patients with DiamondBlackfan anemia. Blood. 2012; 119:2376-2384. 
28. Sloan K, Bohnsack MT, Watkins NJ. The 5S RNP couples p53 homeostasis to ribosome biogenesis and nuclear stress. Cell Report. 2013; 5:237-247.

29. Liao JM, Zhou X, Gatignol A, Lu H. Ribosomal proteins L5 and L11 cooperatively inactivate c-Myc via RNAinduced silencing complex. Oncogene. 2015; 33:49164923.

30. Fancello L, Kampen KR, Hofman IJF, Verbeeck J, De Keersmaecker K. The ribosomal protein gene RPL5 is a haploinsufficient tumor suppressor in multiple cancer types. Oncotarget. 2017; 8:14462-14478.
31. Vander Heiden MG, Cantley LC, Thompson CB. Understanding the Warburg effect: the metabolic requirements of cell proliferation. Science. 2009; 324:1029-1033.

32. Shapiro MB, Senapathy P. RNA splice junctions of different classes of eukaryotes: sequence statistics and functional implications in gene expression. Nucl Acids Res. 1987; 15:7155-7174.

(Received October 21, 2019; Revised December 14, 2019; Accepted December 21, 2019) 